\title{
Análise espacial dos óbitos por tuberculose pulmonar no estado do Amazonas
}

RESUMO | Objetivo: analisar a distribuição dos óbitos por tuberculose pulmonar no estado do Amazonas. Método: trata-se de um estudo ecológico com dados secundários do Sistema de Informação de Mortalidade, durante o período de 2007 a 2017. O software QGIs foi utilizado para elaborar um mapa digital e o software RStudio para as análises estatísticas. Na análise dos dados foi aplicado a regressão linear múltipla, o índice de Moran Global e o teste multiplicador de Lagrange. Resultados: identificou-se 1.267 casos de óbitos por tuberculose pulmonar no estado do Amazonas. A maioria dos óbitos ocorreram no sexo masculino (64,64\%); solteiros (47,43\%); com faixa etária $\geq 60$ anos $(51,14 \%)$; raça/cor parda $(71,11 \%)$. Verificou-se que não existe autocorrelação espacial através do índice de Moran Global (0.0094). Conclusão: A distribuição espacial dos óbitos ocorreu de forma heterogênea nas diferentes regiões do estado Amazonas, apresentando elevadas taxas de mortalidade durante o período de 2007 a 2017.

Palavras-chaves: Tuberculose Pulmonar; Óbito; Distribuição Espacial.

ABSTRACT | Objective: to analyze the distribution of deaths from pulmonary tuberculosis in the state of Amazonas. Method: it is an ecological study with secondary data from the Mortality Information System, during the period from 2007 to 2017. The QGls software was used to create a digital map and the RStudio software for statistical analysis. In the data analysis, multiple linear regression, the Moran Global index and the Lagrange multiplier test were applied. Results: 1,267 cases of deaths from pulmonary tuberculosis were identified in the state of Amazonas. Most deaths occurred in males (64.64\%); singles (47.43\%); aged $\geq 60$ years (51.14\%); race / brown color (71.11\%). It was found that there is no spatial autocorrelation using the Moran Global index (0.0094). Conclusion: The spatial distribution of deaths occurred heterogeneously in different regions of the state of Amazonas, with high mortality rates during the period from 2007 to 2017.

Keywords: Pulmonary Tuberculosis; Death; Spatial Distribution.

RESUMEN | Objetivo: analizar la distribución de muertes por tuberculosis pulmonar en el estado de Amazonas. Método: se trata de un estudio ecológico con datos secundarios del Sistema de Información de Mortalidad, durante el período de 2007 a 2017. Se utilizó el software QGIs para crear un mapa digital y el software RStudio para análisis estadístico. En el análisis de datos se aplicó regresión lineal múltiple, el índice Moran Global y la prueba del multiplicador de Lagrange. Resultados: se identificaron 1.267 casos de defunciones por tuberculosis pulmonar en el estado de Amazonas. La mayoría de las muertes ocurrieron en hombres (64,64\%); solteros (47,43\%); edad $\geq 60$ años (51,14\%); raza / color marrón (71,11\%). Se encontró que no existe autocorrelación espacial usando el índice Moran Global (0.0094). Conclusión: La distribución espacial de las defunciones ocurrió de manera heterogénea en diferentes regiones del estado de Amazonas, con altas tasas de mortalidad durante el período 2007 a 2017.

Palabras claves: Tuberculosis Pulmonar; Muerte; Distribución Espacial.

\section{Mirely Ferreira dos Santos}

Enfermeira - Doutoranda em Saúde Pública pela Universidade de São Paulo - USP. Mestre em Ciências da Saúde pela UFRR. Especialista em Urgência e Emergência (FIP/PB). Docente EBTT do Instituto Federal do Amazonas-IFAM/Atualmente em Exercício Provisório na Reitoria Instituto Federal de São Paulo/IFSP - Coordenadoria de Assistência à Saúde do Servidor - CSS.

ORCID: 0000-0002-8842-3678

\section{Jacinta Ferreira dos Santos Rodrigues}

Professora de Língua Portuguesa. Mestre em Letras pela Universidade Federal de Roraima - UFRR. Especialista em Língua, Linguística e Literatura (FIP/PB). Docente EBTT do Instituto Federal da Paraíba - IFPB. ORCID: 0000-0003-2452-9956

Recebido em: 05/11/2020

Aprovado em: 23/11/2020

\section{Mikaely Ferreira dos Santos}

Administradora pela Universidade Estadual da Paraíba - UEPB. MBA em Coaching pela UNINASSAU - PE.

ORCID: 0000-0001-7319-1440

\section{INTRODUÇÃO}

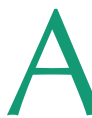
tuberculose (TB) é uma doença infecciosa causada por Mycobacterium tuberculosis ${ }^{1,2}$. É um bacilo álcool-ácido resistente (BAAR) que tem uma preferência por tecido pulmonar, mas também pode afetar outras regiões do corpo, como ossos, nervos, dentre outras. É considerada uma das mais antigas doenças infecciosas da humanidade e, embora seja passível de prevenção e tratamento, permanece na atualidade como um grave problema de saúde pública. Segundo a Organização Mundial de Saúde (OMS), em 2016, ocorreram no mundo 10,4 milhões de casos novos de tuberculose e 1,3 milhões de óbitos ${ }^{1}$.

São vários os fatores que têm sido associados à tuberculose, incluindo fatores endógenos (idade, sexo, raça, presença de HIV e diabetes) e hábitos de vida (consumo de álcool) $)^{3,4}$. Essa abordagem, ainda que identifique fatores de risco em nível individual, não considera o contexto socioeconômico da população, que é um dos principais determinantes da incidência da TB ${ }^{5}$.

A prevalência e a mortalidade por TB vêm sofrendo várias alterações ao longo dos anos no mundo todo, as perspectivas eram que a maioria dos países alcançassem a meta de redução desses coeficientes em $50 \%$ em relação aos indicadores de 1990 até 2015. No ano 2015, um desafio foi lançado pela Organização Mun- 

lose até 20506. A doença representa a segunda causa de óbitos entre as infecciosas e causou, em 2012, cerca de 1,3 milhões de mortes no mundo, demonstrando sua gravidade, especialmente nos 22 países que concentram $80 \%$ da carga da doença' ${ }^{1}$.

O Brasil está entre os 30 países que concentram $80 \%$ dos casos de TB no mundo ${ }^{1}$. Anualmente, são registrados cerca de 73 mil casos novos e 4.500 óbitos por tuberculose no país ${ }^{7}$, sendo o Amazonas, o estado com a maior taxa de incidência de TB, estimada em 71,4 casos por 100 mil habitantes, em 2017, o dobro da incidência média do país que foi de 33,5 casos por 100 mil habitantes ${ }^{8}$.

No período de 2001 a 2011, a taxa de mortalidade média anual no Amazonas foi de 3,5 óbitos por 100 mil habitantes, superior à taxa média do país que foi de 2,5 óbitos por 100 mil habitantes. Nesse período, tanto a taxa de incidência quanto a de mortalidade apresentaram uma dial de Saúde: a eliminação da tubercu-

lenta redução ${ }^{9}$. Nos últimos anos tem se observado o agravamento da doença, com aumento médio anual de 3\% da taxa de incidência ${ }^{10}$.

A análise espacial e temporal tem sido utilizada como importante ferramenta para auxiliar no processo de compreensão da complexidade da tuberculose ${ }^{11}$. Diante do exposto, este estudo teve como objetivo analisar a tendência e distribuição dos óbitos por TB pulmonar no estado do Amazonas, durante o período de 2007 a 2017, e identificar as áreas com maior frequência de óbitos por município.

\section{MÉTODO}

Trata-se de um estudo ecológico com dados secundários do Sistema de Informação de Mortalidade (SIM), sendo a unidade de análise os municípios do estado do Amazonas. A população do estudo foi constituída pelos indivíduos residentes no referido estado, que tiveram diagnóstico de TB pulmonar e evoluíram

\section{Figura 1 - Distribuição dos munícios e regiões de saúde no estado do Amazonas.}

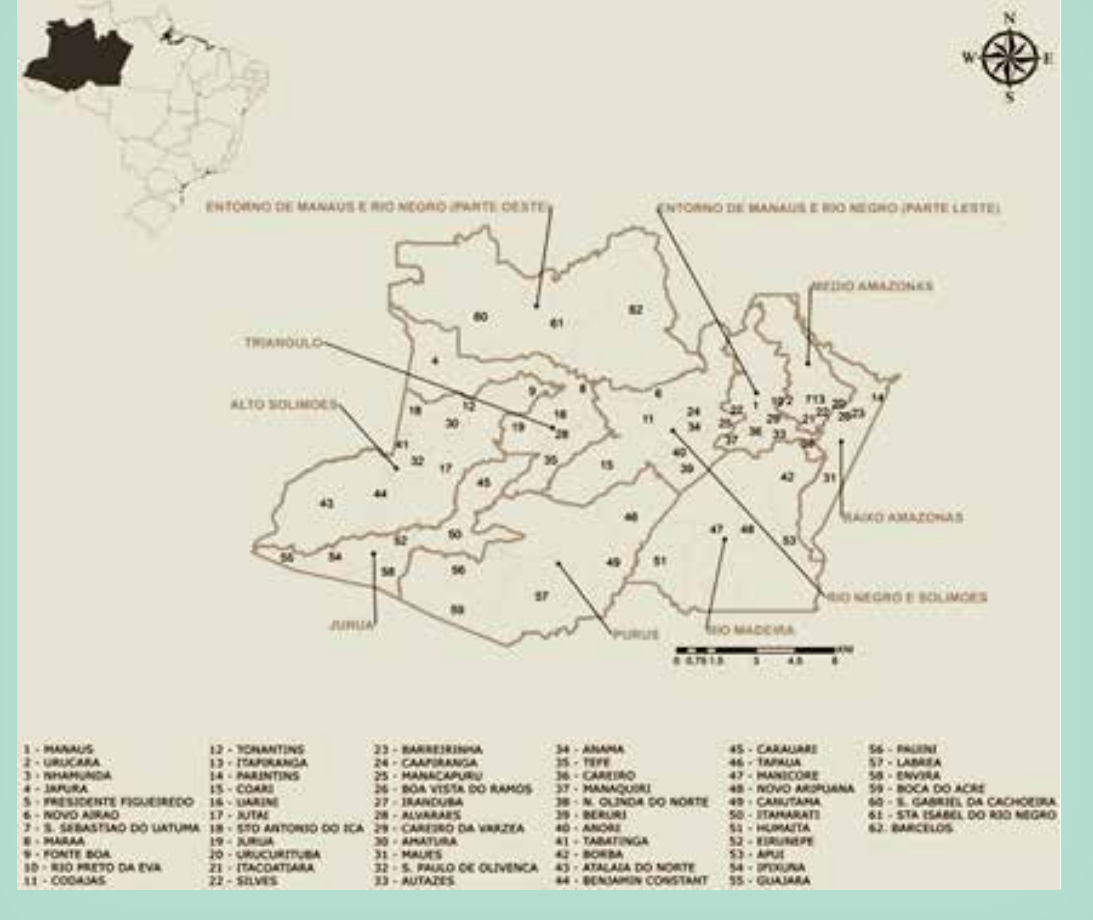

Fonte: IBGE. para óbito no período de 2007 a 2017.

As informações sobre o número de óbitos, conforme os anos 2007 a 2017, foram coletadas a partir de dados secundários disponibilizados no SIM do DATASUS ${ }^{12}$. Já os dados sobre as características sociodemográficas da população dos municípios do estado do Amazonas, foram coletados através dos censos realizados pelo Instituto Brasileiro de Geografia e Estatística (IBGE) nos anos de 2000 e 2010, utilizando o Sistema IBGE de Recuperação Automática - SIDRA ${ }^{13}$.

Segundo o Censo Demográfico do ano 2010, o estado do Amazonas possui uma população de 3.483 .985 habitantes, sendo esse contingente populacional considerado o segundo maior da Região Norte e corresponde a aproximadamente $1,8 \%$ da população atual do Brasil ${ }^{13}$. O Amazonas apresenta área total de 1.559.161 km2, sendo formado por 62 municípios, a capital Manaus é a maior cidade da Região Norte, com 2,1 milhões de habitantes. A figura 1 mostra a distribuição dos municípios e as 9 regiões de saúde no estado do Amazonas.

Nesse estudo, as variáveis de interesse foram o número de óbitos de tuberculose pulmonar ocorridos em cada município no estado do Amazonas durante os anos 2007 a 2017, obtidos através da declaração de óbitos no SIM do DATASUS, tendo como causa básica TB pulmonar - Classificação Internacional de Doenças, $10^{\mathrm{a}}$ revisão (CID - 10). Foi calculado a taxa de mortalidade por 100.000 habitantes, conforme a razão entre o número de óbitos e a população residente. Também foram selecionadas as variáveis: sexo; estado civil; raça/cor; idade; escolaridade e local de ocorrência do óbito.

Após a coleta dos dados foi elaborado um banco de dados no Excel com todas as informações das variáveis do estudo. O software QGls foi utilizado para elaborar um mapa digital com os segmentos dos municípios do estado do Amazonas, disponibilizando, assim, a extensão shapefile. A planilha Excel foi convertida em CSV e exportada para o 
QGIs para ser transformada na tabela de atributos. Posteriormente, esses dados foram transformados em shape para serem trabalhados e analisados no software R, utilizando a linguagem para computação estatística (versão 3.6.1) ${ }^{14}$ no RStudio (versão 1.1.463).

Para a análise dos dados, foi utilizada regressão linear múltipla, a análise exploratória inicial compreendeu a estimativa da ocorrência de óbitos por tuberculose pulmonar com as variáveis sociodemográficas. O índice de Moran Global foi calculado e, também, foi aplicado o teste multiplicador de Lagrange para verificar a existência de dependência espacial. A pesquisa atendeu e respeitou as diretrizes da Resolução 466/2012 do Conselho Nacional de Saúde do Ministério da Saúde ${ }^{15}$, sendo que não necessitou de aprovação do Comitê de Ética de Pesquisa, pois os dados secundários utilizados nesse estudo são de domínio público e não houve identificação dos participantes.

\section{RESULTADOS}

Foram identificados 1.267 casos de óbitos por TB pulmonar no estado do Amazonas. A figura 2 mostra a taxa de

Figura 2 - Caracterização da taxa de mortalidade por tuberculose pulmonar no estado do Amazonas, 2007 a 2017.

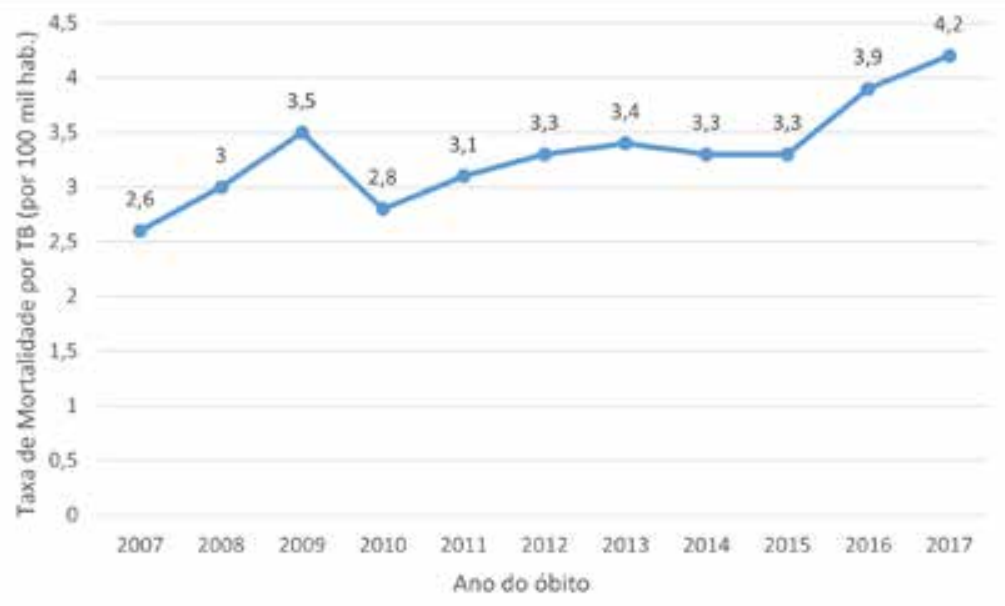

Fonte: SIM e IBGE. mortalidade por TB pulmonar, conforme os anos 2007 a 2017. Percebe-se que nos anos 2007 (2,6 casos para cada 100.000 habitantes) a 2009 (3,5 casos para cada 100.000 habitantes) houve uma tendência de aumento na taxa de mortalidade, já em 2010 (2,8 casos para cada 100.000 habitantes) ocorreu um declínio, seguido de um novo aumento em 2011 (3,1 casos para cada 100.000 habitantes) com tendência crescente até 2017 (4,2 casos para cada 100.000 habitantes).

Os munícipios que apresentaram uma maior proporção de óbitos por TB pulmonar no período de 2007 a 2017, foram: Manaus (59,52\%); São Gabriel da Cachoeira (3\%); Parintins (3\%); Manacapuru (2,8\%); Maués (2,4\%); Tabatinga $(2 \%)$; Itacoatiara $(1,7 \%)$. Todos os 62 municípios do estado apresentaram casos de óbitos, sendo que alguns municípios possuíram registro de apenas um caso, destacam-se Anamã, Guajará, Japurá, Jaruá, Maraã e Tonantis. A letalidade por TB pulmonar no estado do Amazonas foi de 3,88 para cada 100 casos.

No que tange as variáveis sociodemográficas dos indivíduos que foram a óbito no estado do Amazonas, durante o período de 2007 a 2017, identificou-se que a maioria dos óbitos ocorreu em pessoas do sexo masculino $(64,64 \%)$; solteiros $(47,43 \%)$; quanto à idade, a maior proporção possuía uma faixa etária $\geq 60$ anos (51,14\%); a raça/cor mais frequente foi a parda $(71,11 \%)$, seguida por branca $(12,94 \%)$ e indígena (9,94\%). Em relação a variável escolaridade, (25,8\%) não apresentaram informação, seguido de baixa escolaridade (23,6\%). Observou-se também preponderância dos óbitos com ocorrência no ambiente hospitalar $(84,21 \%)$.

$\mathrm{Na}$ análise espacial foram geocodificados os 1.267 casos de óbitos, de acordo com o período estudado. A figura 3 mostra um mapa com a distribuição dos municípios com taxas de mortalidade mais elevadas, conforme os gradientes das cores com tons mais escuros, demonstrando, assim, uma disparidade 
na distribuição espacial dos óbitos no estado do Amazonas. Observa-se que as áreas mais escuras de ocorrência dos óbitos por TB pulmonar estão concentradas nos municípios de Manaus, São Gabriel da Cachoeira, Maués, Tabatinga, Iranduba, Manacapuru, Itacoatiara, Tefé e Parintins.

Através da aplicação da regressão linear múltipla, identificou-se que existe colinearidade entre as variáveis independentes (sexo; estado civil; raça/cor; idade; escolaridade). Todas as pressuposições foram verificadas nos resíduos. Os resíduos em função dos valores preditos apresentaram distribuição normal e em função das coovariáveis, observou-se que existe independência dos resíduos.

$O$ índice de Moran Global foi calculado e conforme resultado obtido (0.0094), verificou-se que não existe autocorrelação espacial, pois seu valor foi baixo. Para ter certeza da inexistência de dependência espacial, foi aplicado o teste multiplicador de Lagrange (LMlag = 0.0387 e $p<0.8439$ ), o mesmo confirmou que não existe dependência espacial, pois os valores da estatística do teste foram muito baixos.

\section{Figura 3 - Distribuição dos casos de óbitos por tuberculose pulmonar, segundo} município de residência, estado Amazonas, de 2007 a 2017.

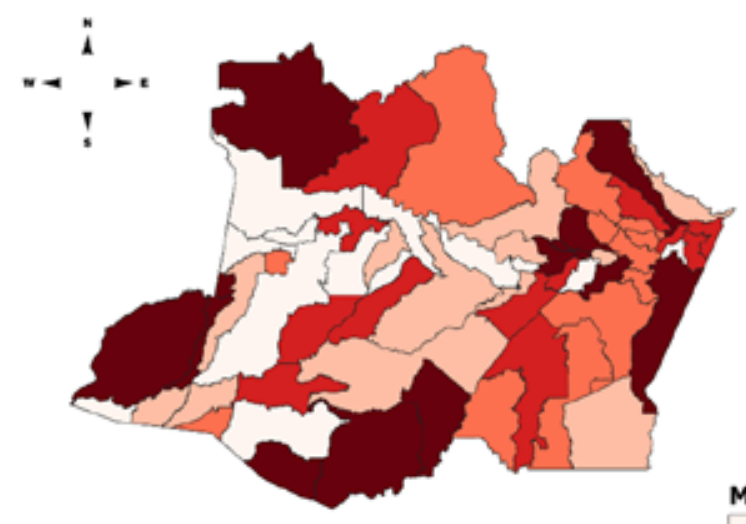

Mun_AM

प5,7052-15,4022

$\square 15,4022-21,0878$

$\square 21,0878 \cdot 26,2025$

- $26,2025 \cdot 40,4059$

- $40,4059 \cdot 100,2744$
DISCUSSÃO

As taxas de mortalidade por TB pulmonar identificadas nesse estudo foram significativamente altas no estado do Amazonas durante o período de 2007 a 2017, sendo constatado que a cidade de Manaus apresentou a maior proporção de óbitos no estado. Esses dados são preocupantes, sabendo que a TB pulmonar é uma doença curável e que o tratamento é oferecido de forma gratuita pelo Sistema Único de Saúde-SUS. Esses resultados são discrepantes quando comparamos com outras regiões e estados do Brasil, em que as taxas de mortalidade são relativamente mais baixas ${ }^{16}$.

Estudos relatam que a TB é uma doença infecciosa transmissível, por vias aéreas, que anualmente acomete milhares de pessoas. A multirresistência as drogas e a coinfecção pelo HIV dificultam mais ainda o tratamento. Embora esse seja oferecido gratuitamente pelo Ministério da Saúde, ainda há elevadas taxas de incidências, e evoluções para óbitos decorrentes da TB, essas consequências podem ser atribuídas ao abandono do tratamento ou até mesmo a adesão inadequada ${ }^{17}$.

Os estados brasileiros são marcadamente heterogêneos, tanto no que se refere aos indicadores de morbimortalidade por TB, quanto os indicadores de desempenho das ações de controle, sendo que os estados das regiões Norte e Nordeste apresentam os piores índices, no que tange à carga da doença e ao desempenho das medidas de controle ${ }^{7}$.

No tocante as variáveis sociodemográficas analisadas, identificou-se que a maioria dos casos de óbitos eram do sexo masculino, solteiros, com idade $\geq$ a 60 anos. Esses resultados são consistentes com o estudo realizado no Rio Grande Norte, que relata uma maior proporção de óbitos por TB pulmonar em homens ${ }^{18}$. Quanto a idade, diferem com os resultados das pesquisas realizadas nos estados de Minas Gerais ${ }^{19}$, Mato Grosso do Sul ${ }^{20}$ e Ceará21, que afirmam que os óbitos ocorreram em maior quantidade entre 
pessoas com idade $\leq 52$ anos. Já a variável estado civil, corrobora com os resultados das pesquisas acima, demonstrando prevalência de solteiros ${ }^{19,20,21}$.

No que se refere a raça/cor, o estudo mostrou que a maioria dos casos $(71,11 \%)$ foram pardos. Este resultado é consistente com dados de pesquisas no Maranhão $(68,91 \%)^{22}$ e em Mato Grosso do Sul $(50,5 \%)^{20}$ que demonstraram que a maior parte dos óbitos ocorreu em indivíduos de raça/cor parda. Quanto a escolaridade, constatou-se predominância de baixa escolaridade, esses resultados expressam similaridade com estudos que demonstraram prevalência do analfabetismo ou a baixa escolaridade como situações menos privilegiadas do ponto de vista financeiro, expressando assim fatores de risco para a TB pulmonar ${ }^{19,20,23}$.

Outra pesquisa também relata que a baixa escolaridade, o desemprego e a renda são fatores individuais associados ao aumento da incidência de TB e à baixa adesão ao tratamento, podendo estar relacionados ao acesso aos serviços de saúde e à qualidade do diagnóstico. As pessoas com menos educação e menores rendimentos são menos propensas a perceber que estão em risco e a cumprir o tratamento, pois possuem desigualdade no acesso individual à informação, aos benefícios decorrentes do conhecimento, aos bens de consumo e principalmente aos serviços de saúde ${ }^{24}$.

Com relação a variável local de ocorrência do óbito, referente ao perfil operacional, foi identificado que a maior proporção dos óbitos ocorreu no ambiente hospitalar. Pode-se corroborar esses achados com estudos nacionais realizados nos estados no Rio Grande do Nor$\mathrm{te}^{18}$, Maranhão ${ }^{22}$, Mato Grosso do $\mathrm{Sul}^{23}$ e no município de São Paulo ${ }^{25}$, os quais apresentaram registros superiores a $80 \%$ de óbitos por TB pulmonar ocorridos no hospital.

A análise espacial permitiu identificar a distribuição dos casos de óbitos por TB pulmonar, sendo mais frequentes em determinados municípios no estado do Amazonas. Através da inspeção do mapa de distribuição espacial (Figura 3), evidenciou-se disparidades espaciais com distribuição de mortes por TB pulmonar de forma mais intensa nas regiões em Entorno de Manaus; Rio Negro (oeste); Rio Juruá; nas regiões do Médio e Baixo Amazonas e no Alto Solimões. Esses resultados são importantes para identificar o padrão de distribuição desse agravo a saúde da população no estado do Amazonas.

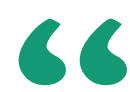

\section{A análise espacial permitiu identificar a distribuição dos casos de óbitos por TB pulmonar, sendo mais frequentes em determinados municípios no estado do Amazonas.}

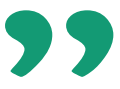

Nota-se que algumas dessas regiões, onde a mortalidade por TB pulmonar foi maior, como no Rio Negro (oeste do estado do Amazonas), o Médio e o Baixo Amazonas, coincidem com os municípios que apresentam baixas condições sociais com áreas de alta vulnerabilidade social, como por exemplo, o município de São Gabriel da Cachoeira. Esses dados mostram que os fatores socioeconômicos desempenham um papel importante na magnitude da TB.

A capital Manaus apresentou uma proporção de óbitos expressivamente alta quando comparada com os demais municípios no estado do Amazonas, como grande parte da população amazonense reside em Manaus e, sabendo que esta cidade teve um grande aumento populacional na última década, devido ao processo migratório, principalmente de indígenas em busca de melhores condições de vida, consequentemente, isso repercutiu em uma concentração maior no número de aglomerados subnormais.

O comportamento da TB, como o de outras endemias, é fortemente influenciado pelo meio, sendo possível evidenciar que a associação entre TB e precárias condições socioeconômicas data dos primórdios da epidemiologia dessa doença, havendo, assim, a necessidade de estudá-la e sobre ela intervir, levando-se em consideração sua distribuição espacial ${ }^{26}$. A análise espacial é relevante na investigação e compreensão da ocorrência e distribuição da mortalidade em uma cidade, pois é o ambiente onde circula o agente infeccioso que, em condições específicas, provoca a doença e, até mesmo a morte em decorrência dela ${ }^{27}$.

Estudos demonstram que as estratégias para que ocorra o alcance das metas globais pactuadas, se faz necessário integrar sistematicamente um conjunto de ações de vigilância, que identifique os pontos principais entre o processo de captação e notificação dos casos novos da doença ${ }^{8}$. A informação em saúde deve ter como finalidade reduzir as incertezas e a identificação de situações prioritárias com vistas a subsidiar um planejamento adequado para a execução de ações que condicionem a realidade às transformações necessárias ${ }^{28}$.

\section{CONCLUSÃO}

A distribuição espacial dos óbitos 
por tuberculose pulmonar ocorreu de forma heterogênea nas diferentes regiões no estado do Amazonas, estabelecendo consistência com as condições socioeconômicas da população. O presente estudo traz informações de elevadas taxas de mortalidade durante o período de 2007 a 2017, esses achados demonstram que a TB pulmonar ainda consiste como um problema de saúde pública no estado do Amazonas e que são necessárias novas estratégias para enfrentamento dessa doença. Os resultados da pesquisa também poderão contribuir na orientação de gestores e profissionais de saúde quanto as áreas prioritárias da ocorrência de TB pulmonar e na tomada de decisões para a efetividade de políticas públicas e estratégias de promoção da saúde.

\section{Referências}

1. Who. Global tuberculosis report 2017. World Health Organization. Geneva: 2017.

2. Brasil. Portal da Saúde. Ministério da Saúde. Tuberculose. Brasília: Ministério da Saúde, 2017. [Acesso em: 25 de ago. 2020]. Disponível em: https://portalarquivos2.saude.gov.br/images/pdf/2017/junho/29/plano_nacional_tb_web.pdf 3. Lacerda SNB, Temoteo RCA, Figueiredo TMRM, Luna FDT, Sousa MAN, Abreu LC, Fonseca FLA. Individual and social vulnerabilities upon acquiring tuberculosis: a literature systematic review. Int Arch Med. 2014; 7:1-8. [Acesso em: 28 de ago. 2020]. Disponível em: https://doi.org/10.1186/1755-7682-7-1

4. Ferrara G, Murray M, Winthrop K, Centis R, Sotgiu G, Migliori GB, Maeurer M, Zumla A. Risk factors associated with pulmonary tuberculosis: smoking diabetes and anti-TNF囚 drugs. Curr Opin Pulm Med. 2012; 18:233-40. [Acesso em: 11 de out. 2020]. Disponível em: https://doi.org/10.1097/MCP.0b013e$328351 \mathrm{fgd} 6$

5. Lonnroth K, Castro KG, Chakaya JM, Chauhan LS, Floyd K, Glaziou P, Raviglione MC. Tuberculosis control and elimination 2010-50: cure, care, and social development. Lancet. 2010; 375:1814-29. [Acesso em: 11 de out. 2020]. Disponivel em: https://doi.org/ 10.1016/S0140-6736(10)60483-7

6. Who. The global plan to stop TB 2011-2015: transforming the fight towards elimination of tuberculosis - reprinted with changes, 2011. World Health Organization. Geneva: 2011.

7. Brasil. Boletim Epidemiológico. Ministério da Saúde. Brasília: 2015.

8. Brasil. Boletim epidemiológico - Implantação do Plano Nacional pelo Fim da Tuberculose como Problema de Saúde Pública no Brasil: primeiros passos rumo ao alcance das metas. Secretaria de Vigilância em Saúde/ Ministério da Saúde. Brasília. 2018.

9. GARRIDO MS, Sékula SB, Souza AB, et al. Temporal distribution of tuberculosis in the State of Amazonas, Brazil. Revista da Sociedade Brasileira de Medicina Tropical. 2015; v. 48, p. 63-69.

10. Castro DB. Tuberculose na população amazonense: distribuição espacial, determinantes sociais e a desigualdade da incidência nos anos recentes [tese]. Rio de Janeiro: Fundação Oswaldo Cruz, Escola Nacional de Saúde Pública Sergio Arouca; 2018. [Acesso em: 16 de out. 2020]. Disponível em: https:/l www.arca.fiocruz.br/bitstream/icict/30886/2/daniel_barros_de.pdf

11. Santos FGB, Paiva JPS, Araújo EMCF, Leal TC, Souza CDF, Duailibe FT. Tuberculose no estado de Alagoas: análise espacial e temporal entre 2010 e 2015 0 Mundo da Saúde, São Paulo. 2019; 43 (1): 129-150. [Acesso em: 28 de ago. 2020]. Disponível em: 10.15343/0104-7809.20194301129150

12. Brasil, Ministério da Saúde. SIM/DATASUS. Sistema de Informação sobre Mortalidade. [Acesso em: 26 de mai. 2020]. Disponível em: http://tabnet.datasus.gov.br/cgi/deftohtm.exe?sim/cnv/obt10am.def

13. IBGE, Instituto Brasileiro de Geografia e Estatística. [Acesso em: 29 de ago. 2020]. Disponivel em: https://sidra.ibge.gov.br/pesquisa/censo-demografico/ demografico-2010/universo-caracteristicas-da-populacao-e-dos-domicilios

14. R Equipe Central, 2018. R: uma linguagem e ambiente para computação estatística. Viena, Áustria: R Foundation for Statistical Computing. Disponível em: https://www.R-project.org/

15. Brasil. Ministério da Saúde. Comissão Nacional de Ética em Pesquisa. Conselho Nacional de Saúde (BR). Diretrizes e Normas regulamentadoras de pesquisa envolvendo seres humanos. Resolução $N^{\circ} 466 / 12$ de 12 de dezembro de 2012 - CNS. Brasília, DF, 2012.

16. Brasil, Ministério da Saúde. Sala de Apoio à Gestão Estratégica. Brasília:
SAGE/SUS [Acesso em: 10 de ago. 2020]. Disponível em: http://189.28.128.178/ sagel

17. Sousa LO, Mitano F, Lima MCRAA, Sicsú AN, Silva LMC, Palha PF. Terapia de curta duração da tuberculose: uma análise discursiva. Rev Bras Enferm. 2016; 69 (6):1154 - 63. [Acesso em: 16 de out. 2020]. Disponível em: http://dx.doi. org/10.1590/0034-7167-2016-0330

18. Queiroz AAR, Berra TZ, Garcia MCC, Popolin MP, Belchior AS, Yamamura M et al. Padrão espacial e tendência temporal da mortalidade por tuberculose. Rev. Latino-Am. Enfermagem. 2018; 26 - 2992. [Acesso em: 16 de out. 2020]. Disponível em: http://10.1590/1518-8345.2049.2992

19. Augusto CJ, Carvalho WS, Gonçalves AD, Ceccato MG, Miranda SS. Characteristics of tuberculosis in the state of Minas Gerais, Brazil: 2002-2009. J Bras Pneumol. 2013; 39(3):357-64. [Acesso em: 16 de out. 2020]. Disponível em: http://dx.doi.org/10.1590/ S1806-37132013000300013

20. Espindola LCD. Estudo da mortalidade por tuberculose em Campo Grande - MS, 2001 a 2008 [Dissertação]. Rio de Janeiro: Escola Nacional de Saúde Pública; 2010. [Acesso em: 20 de out. 2020]. Disponível em: https://www.arca. fiocruz.br/bitstream/icict/23063/1/1159.pdf

21. Façanha MC. Evolução da mortalidade por tuberculose em Fortaleza (CE), entre 1980 e 2001. J Bras Pneumol. 2006; 32(6): 553-8. [Acesso em: 11 de out. 2020]. Disponível em: http://dx.doi.org/10.1590/ S1806-37132006000600013 22. Santos M, Yamamura M, Garcia MCC, Popolin MP, Silveira TRS, Arcêncio RA. Análise espacial dos óbitos por tuberculose pulmonar em São Luís, Maranhão. J Bras Pneumol. 2014; 40 (5): 543 - 551. [Acesso em: 10 de ago. 2020]. Disponível em: http://dx.doi.org/10.1590/S1806-37132014000500011

23. Larroque MM, Pontes ERJC, Marques APC, Fernandes SM. Mortalidade por tuberculose em municípios prioritários do estado de Mato Grosso do Sul, 1999-2008. Arq. Ciênc. Saúde UNIPAR, Umuarama. 2013; 17 (3): 163-169. [Acesso em: 10 de ago. 2020]. Disponivel em: https://revistas.unipar.br/index. php/saude/article/viewFile/5066/2948

24. Yamamura M, Santos-Neto M, Santos RAN, Garcia MCC, Nogueira JÁ, Arcêncio RA. Epidemiological characteristics of cases of death from tuberculosis and vulnerable territories. Rev. Latino-Am. Enfermagem. 2015; 23 (5): 910-8. [Acesso em: 11 de out. 2020]. Disponível em: http://dx.doi.org/10.1590/01041169.0450 .2631

25. Lindoso AA, Waldman EA, Komatsu NK, Figueiredo SM, Taniguchi M, Rodrigues LC. Profile of tuberculosis patients progressing to death, city of São Paulo, Brazil, 2002. Rev Saúde Pública. 2008; 42 (5): 805-12. [Acesso em: 16 de out. 2020]. Disponivel em: http:// dx.doi.org/10.1590/S0034-89102008000500004 26. Hino P, da Costa-Junior ML, Sassaki CM, Oliveira MF, Villa TC, dos Santos CB. Time series of tuberculosis mortality in Brazil (1980-2001). Rev Lat Am Enfermagem. 2007;15 (5): 936-41. [Acesso em: 16 de out. 2020]. Disponível em: http://dx.doi.org/10.1590/ S0104-11692007000500009

27. Maciel ENL. Post-2015 agenda strategies for tuberculosis control in Brazil: challenges and opportunities. Epidemiol Serv Saúde. 2016. [Acesso em: 28 de ago. 2020]. Disponível em: http://dx.doi. org/10.5123/s167949742016000200021

28. Souza MSPL, Aquino R, Pereira SM, Costa MCN, Barreto ML, Natividade $M$ et al. Fatores associados ao acesso geográfico aos serviços de saúde por pessoas com tuberculose em três capitais do Nordeste brasileiro. Cad. Saúde Pública. 2015; 31(1): 111-120. 